\title{
Dynamics of ventricular repolarisation in the congenital long QT syndromes
}

\author{
Nicholas J Linker, A John Camm, David E Ward
}

\begin{abstract}
Patients with congenital QT interval prolongation are at risk of ventricular arrhythmias and sudden death. It has been suggested that the susceptibility to arrhythmias in these syndromes may be related to the abnormal dynamics of ventricular repolarisation. The dynamics of ventricular repolarisation, including assessment of the effect of changing heart rate on the QT interval and the duration of the right ventricular monophasic action potential, were studied in eight patients with congenital long QT syndromes. The effects of altered sympathetic tone on these dynamics were investigated with isoprenaline, propranolol, and left stellate ganglion block. The rate adaptation of the QT interval was abnormal in only a few patients and in some patients this feature may be related to the severity of the condition. These abnormalities may be exaggerated by isoprenaline and lessened by propranolol and left stellate ganglion block. Monophasic action potential dynamics were normal in all patients.

The hypothesis that impaired QT rate adaptation may play a role in the genesis of ventricular arrhythmias in these syndromes is not, in general, supported by the present data. However, in patients with impaired adaptation the normalisation of QT dynamics after $\beta$ blockade and left stellate ganglion block was consistent with the efficacy of these forms of treatment.
\end{abstract}

The QT interval of the electrocardiogram reflects the total duration of electrical activity in the ventricles. Two syndromes are associated with a primary hereditary prolongation of the QT interval. In both the Jervell and Lange-Nielsen and the Romano Ward syndromes there is usually a family history of

Table 1 Patient characteristics

\begin{tabular}{llllll}
\hline Patient & Sex & Age & Diagnosis & Symptoms & $\begin{array}{l}\text { Documented } \\
\text { torsade de pointes }\end{array}$ \\
\hline 1 & F & $17 \mathrm{yr}$ & RW & Syncope $\times 3$ & + \\
2 & M & $15 \mathrm{mnth}$ & JLN & Syncope $>300$ & + \\
3 & M & $3 \mathrm{yr}$ & JLN & Syncope $\times 15$ & + \\
4 & F & $7 \mathrm{yr}$ & JLN & Syncope $\times 4$ & + \\
5 & N & $18 \mathrm{mnth}$ & JLN & None & + \\
6 & F & $19 \mathrm{yr}$ & RW & Syncope $\times 1$ & + \\
7 & F & $20 \mathrm{yr}$ & RW & Syncope $\times 3$ & + \\
8 & F & $43 \mathrm{yr}$ & RW & Syncope $>20$ & + \\
\hline
\end{tabular}

RW, Romano Ward syndrome; JLN, Jervell Lange-Nielsen syndrome. syncopal episodes caused by ventricular tachycardia, the most common of which is torsade de pointes, and a family history of sudden cardiac death. ${ }^{1-3}$ It has been suggested that the susceptibility to arrhythmias in these syndromes may be related to an absence or alteration of the normal QT shortening in response to an increase in heart rate. ${ }^{4} \mathrm{We}$ studied the dynamics of ventricular repolarisation, as measured by the QT interval and ventricular monophasic action potential duration, in patients with congenital QT interval prolongation and assessed the effects on these dynamics of alterations in sympathetic tone.

\section{Patients and methods}

We studied eight patients (three male, five female), mean age 14 years (range 15 months to 43 years) (table 1). Four patients (numbers 2 to 5) had sensorineural deafness consistent with the Jervell and Lange-Nielsen syndrome; patients 2 and 3 came from the same family as did patients 4 and 5. Patient 1 had two sisters who died suddenly aged 16 and 20 years, both with a history of syncope. Patients 6,7 , and 8 had no family history of syncope or sudden death. Patient 5 (brother of patient 4) was found to have a long QT interval on routine electrocardiographic screening. Two patients (6 and 8 ) had previously been diagnosed as having either hysterical or epileptiform collapses on several occasions. Seven patients had documented torsade de pointes at the time of syncope.

No patient was taking any antiarrhythmic medication (including $\beta$ blocking agents) at the time of the study. Table 2 gives QRS durations, QT intervals, QTc values, and cycle lengths in sinus rhythm. Four electrocardiographic leads ( $\mathrm{I}, \mathrm{aVF}, \mathrm{V} 1$, and V6) were recorded at paper speeds of 50 or $100 \mathrm{~mm} / \mathrm{s}$. This configuration was preset by the recorder and remained constant throughout the study. The QT interval was measured from lead $V 1$, because this provides a close approximation to the maximum QT interval in any lead. ${ }^{5}$ During sinus rhythm and atrial pacing the QT interval was measured from the start of the QRS complex to the end of the $T$ wave. During ventricular pacing the $Q T$ interval was measured from the ventricular pacing artefact or the beginning of the paced QRS complex (if there was stimulus latency) to the end of the $T$ wave. The end of the $T$ wave was defined as the point of return of the $T$ wave to baseline. ${ }^{6}$ There is no accepted definition of $U$ waves in the long QT syndromes. In the presence of bizarre and often 
Table $2 Q R S$ duration, $Q T$ interval, and $Q T c$ values in all patients before and after propranolol

\begin{tabular}{|c|c|c|c|c|c|c|c|}
\hline \multirow[b]{2}{*}{ Patient } & \multirow{2}{*}{$\begin{array}{l}\text { QRS duration } \\
\text { (sinus rhythm) } \\
\text { (ms) }\end{array}$} & \multicolumn{3}{|c|}{ Baseline (sinus rhythm) } & \multicolumn{3}{|c|}{ Propranolol (sinus rhythm) } \\
\hline & & $\begin{array}{l}Q T \\
(m s)\end{array}$ & $\begin{array}{l}\text { Cycle length } \\
\text { (ms) }\end{array}$ & $\begin{array}{l}Q T c \\
\left(s^{1 / 2}\right)\end{array}$ & $\begin{array}{l}\overline{Q T} \\
(m s)\end{array}$ & $\begin{array}{l}\text { Cycle length } \\
\text { (ms) }\end{array}$ & $\begin{array}{l}Q T c \\
\left(s^{1 / 2}\right)\end{array}$ \\
\hline $\begin{array}{l}1 \\
2 \\
3 \\
4 \\
5 \\
6 \\
7 \\
7 \\
8\end{array}$ & $\begin{array}{r}90 \\
80 \\
90 \\
80 \\
85 \\
90 \\
90 \\
110\end{array}$ & $\begin{array}{l}500 \\
645 \\
440 \\
580 \\
360 \\
500 \\
360 \\
470\end{array}$ & $\begin{array}{l}930 \\
600 \\
680 \\
680 \\
560 \\
950 \\
480 \\
720\end{array}$ & $\begin{array}{l}0.520 \\
0.835 \\
0.535 \\
0.705 \\
0.480 \\
0.515 \\
0.520 \\
0.555\end{array}$ & $\begin{array}{l}480 \\
380 \\
435 \\
520 \\
360 \\
520 \\
390 \\
505\end{array}$ & $\begin{array}{l}980 \\
600 \\
680 \\
810 \\
560 \\
950 \\
650 \\
810\end{array}$ & $\begin{array}{l}0.485 \\
0.490 \\
0.530 \\
0.580 \\
0.480 \\
0.535 \\
0.485 \\
0.560\end{array}$ \\
\hline
\end{tabular}

changing $T$ wave configuration it is not possible clearly to distinguish between the $T$ and $U$ waves. Thus all our measurements were made to the end of total repolarisation as defined above. During ventricular pacing the $T$ wave was always inscribed as a simple curve with no $\mathrm{U}$ waves or multiphasic components.

The QTc values were calculated from the formula adapted by Taran and Szilagyi ${ }^{7}$ from a formula devised by Bazett. ${ }^{8}$ Dimensional analysis of the Taran and Szilagyi formula shows the units of QTc to be $s^{1 / 2} .9$

The procedure was performed after the patient or a parent had given informed consent to an electrophysiology study. In all patients a 6 French gauge bipolar pacing electrode was inserted via the right femoral vein and positioned at the right ventricular apex to permit ventricular pacing. In five patients ( 1 and 4 to 7 ), a 7 French gauge silver/silver chloride monophasic action potential contact electrode was introduced via the right femoral vein and positioned at the right ventricular apex. In one patient (case 1) a monophasic action potential electrode was also positioned at the left ventricular apex via the right femoral artery. In one patient (case 6) a monophasic action potential electrode and a pacing electrode were positioned in the right atrium to allow atrial measurements to be made. The signals from the monophasic action potential electrodes were processed by a custom built AC amplifier with a band width of 0.025 to $500 \mathrm{~Hz}(-3 \mathrm{~dB})$ and recorded on a SiemensElema Mingograf 82 chart recorder at a speed of 50 or $100 \mathrm{~mm} / \mathrm{s}$ together with four surface leads (leads I, aVF, V1, and V6). Pacing was performed with a Digitimer 4279 programmable stimulator. All measurements were made by two observers. The duration of the monophasic action potential was measured at $90 \%$ repolarisation (MAP ${ }_{90}$ ) because it is difficult to determine the exact point of full repolarisation.

The QT interval and $\mathrm{MAP}_{90}$ duration were determined during sinus rhythm in all patients. In all patients ventricular pacing was performed for 10 seconds from the right ventricular apex at a cycle length that was $50 \mathrm{~ms}$ shorter than the intrinsic cycle length of the patient. The QT interval and $\mathrm{MAP}_{90}$ duration of the last paced beat were measured (fig 1). After a 30 second pause, the pacing train was repeated with the cycle length 20 ms shorter; the pacing trains were repeated at shorter cycle lengths until ventricular exit block occurred. All cycles with extrasystoles were discarded and repeated. Three patients $(4,6$, and 8) received an infusion of isoprenaline at $4 \mu \mathrm{g} / \mathrm{kg} / \mathrm{min}$ and in two of these (6 and 8) the protocol was repeated. The protocol was repeated 10 minutes after the injection of $0.2 \mathrm{mg} / \mathrm{kg}$ of propranolol in six patients and in one patient (case 8 who had asthma), after $5 \mathrm{mg}$ of intravenous atenolol. In two patients ( 3 and 4 ) the protocol was repeated after left stellate ganglionectomy (including intravenous propranolol in patient 3) and in one patient (case 2) the protocol was repeated after a marcaine induced left stellate ganglion block.

In patient 1 the recordings were made from both right and left ventricles. In patient 6 the baseline protocol was performed with atrial pacing and recording atrial monophasic action potentials in addition to ventricular studies. In patients 6 and 8 the dispersion of right ventricular $\mathrm{MAP}_{90}$ durations was determined by recording monophasic action potentials from four and six right ventricular sites respectively.

It was not possible to perform every protocol in all patients because this would have needed prolonged studies in very young children. In one patient who received isoprenaline (case 4) spontaneous torsade de pointes occurred repeatedly and the protocol was not completed. Only two patients underwent left stellate ganglionectomy and one left stellate ganglion block. The dispersion of monophasic action potentials was not routinely measured because this has been published previously. ${ }^{10}$ The measurement of atrial monophasic action potentials was introduced late in the study as part of another continuing protocol.

Where appropriate all results were compared by a Student's paired $t$ test.

\section{Results}

QT INTERVAL IN SINUS RHYTHM

All patients had a normal baseline QRS duration (mean (SD) 89 (9) ms) with a mean QT interval of 482 (99) ms at a mean cycle length of 700 (166) ms (90 (20) beats/minute) and an increased QTc $\left(0.583(0.012) \mathrm{s}^{1 / 2}\right)$ (table 2). After $\beta$ blockade with propranolol or atenolol there was no significant change in the mean QT interval (449 (66) $\mathrm{ms})$, QTc value $(0.518$ $\left.(0.038) \mathrm{s}^{1 / 2}\right)$, or cycle length $(755(157) \mathrm{ms}, 90$ (20) beats/minute). Three patients were given isoprenaline (cases 4,6 , and 8 ), after which there was a significant shortening of the QT 
Figure 1 Recording of monophasic action potentials and $Q T$ interval during ventricular pacing in patient 4. For explanation of measurement see text. $R A 1, R A 2$, right atrial electrograms; $R V$, right ventricular electrogram; $M A P$, right ventricular monophasic action potential recording. Paper speed $100 \mathrm{~mm} / \mathrm{s}$.

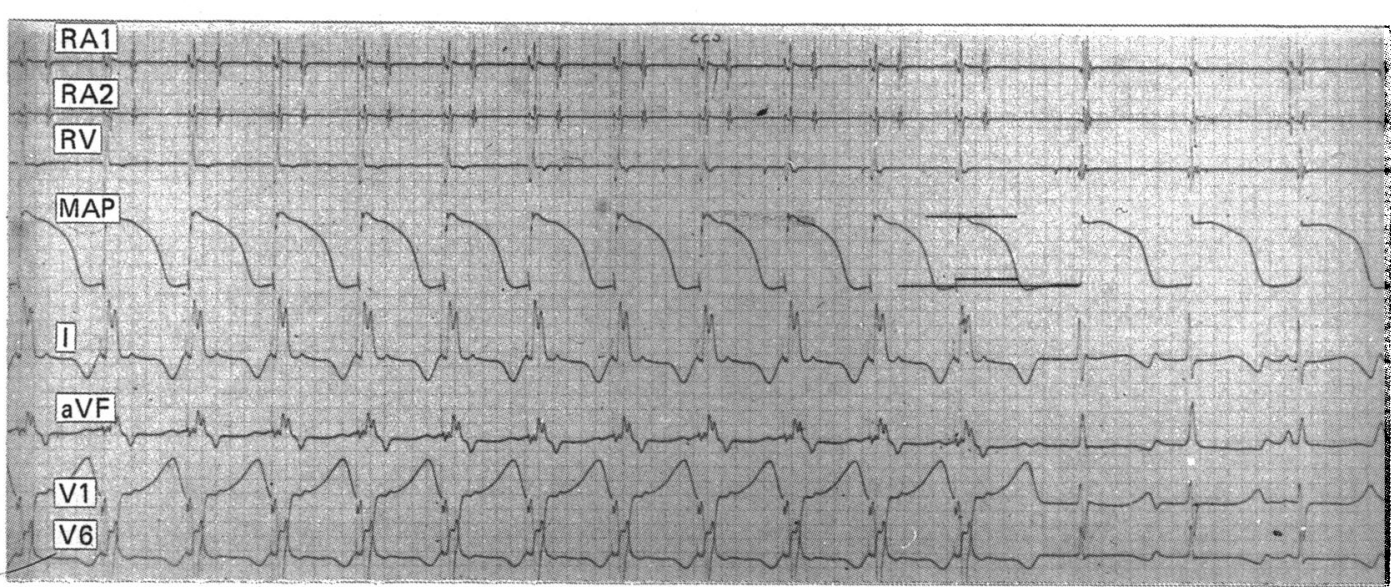

interval (330 (56) ms p $<0.05$ ), though this was in the presence of a shorter cycle length (438 (91) ms, $\mathrm{p}=\mathrm{Ns}$ ) and there was no significant change in the QTc value $(0.498$ $\left.(0.032) \mathrm{s}^{1 / 2}\right)$. In the two patients (cases 3 and 4 ) in whom left stellate ganglionectomy was performed there was no difference between values before and after ganglionectomy in the QT interval (440 and $580 \mathrm{~ms} v 460$ and $580 \mathrm{~ms}$ ), cycle length (680 and $560 \mathrm{~ms} v 560$ and $780 \mathrm{~ms})$, or QTc value $\left(0.535\right.$ and $0.705 \mathrm{~s}^{1 / 2} v$ 0.615 and $0.665 \mathrm{~s}^{1 / 2}$ ).

\section{MONOPHASIC ACTION POTENTIALS IN SINUS RHYTHM}

In the patients in whom monophasic action potentials were recorded (cases 1 and 4 to 8 ) the baseline $\mathrm{MAP}_{90}$ duration was 363 (79) ms. Abnormalities in the configuration of monophasic action potentials, in particular "humps" on phase 3 of the monophasic action potential, were seen in three patients (cases 4,7 , and 8 ). In one patient (case 4) a spontaneous episode of torsades de pointe was recorded during the isoprenaline infusion, which showed that the first beat of the arrhythmia was not related to the "hump" (fig 2). After propranolol there was no significant change in MAP $_{90}$ duration (353 (52) $\mathrm{ms}$ ). In the three patients who received isoprenaline (cases 4,6 , and 8), there was a significant shortening in $\mathrm{MAP}_{90}$ duration (252
(51) ms, p < 0.05), though this was accompanied by a decrease in the sinus cycle length.

\section{QT RATE ADAPTATION}

The response of the patients' QT intervals to the ventricular pacing protocol was as follows. As the pacing rate was increased, there was a tendency for the QT interval to shorten (fig 3), though there was considerable variation between patients. In particular, patients 1 and 2 showed no adaptation of the QT interval to the faster pacing rates. In patient 1 (fig 3 ) the QT interval at a cycle length of $680 \mathrm{~ms}(540 \mathrm{~ms})$ was shorter than at a pacing rate of $400 \mathrm{~ms}$ $(580 \mathrm{~ms})$. In patient 2 there appeared to be no rate adaptation, with the QT interval behaving in a haphazard fashion. Although the other patients tended to shorten their QT interval with decreasing pacing rate, the amount of decrease in QT interval was variable. For example, patient 8 showed very little change (100 ms decrease in QT interval over a pacing range of 600 to $260 \mathrm{~ms}$ ) whereas patient 4 showed a $190 \mathrm{~ms}$ decrease in QT interval over the same pacing range.

In the two patients who received isoprenaline and had the protocol repeated, one (patient 8) showed no change in QT interval duration or dynamics. The other (patient 6) had normal baseline QT dynamics and a total loss of QT adaptation during the isoprenaline infusion
Figure 2 Spontaneous torsade de pointes in patient 4 during an isoprenaline infusion. The initiating beat of the arrhythmia occurs before the right ventricular monophasic action potential, suggesting that the abnormalities in the configuration of the monophasic action potential are not relevant to the initiation of the tachycardia. For further discussion see text. MAP, right ventricular monophasic action potential recording. Paper speed $50 \mathrm{~mm} / \mathrm{s}$.

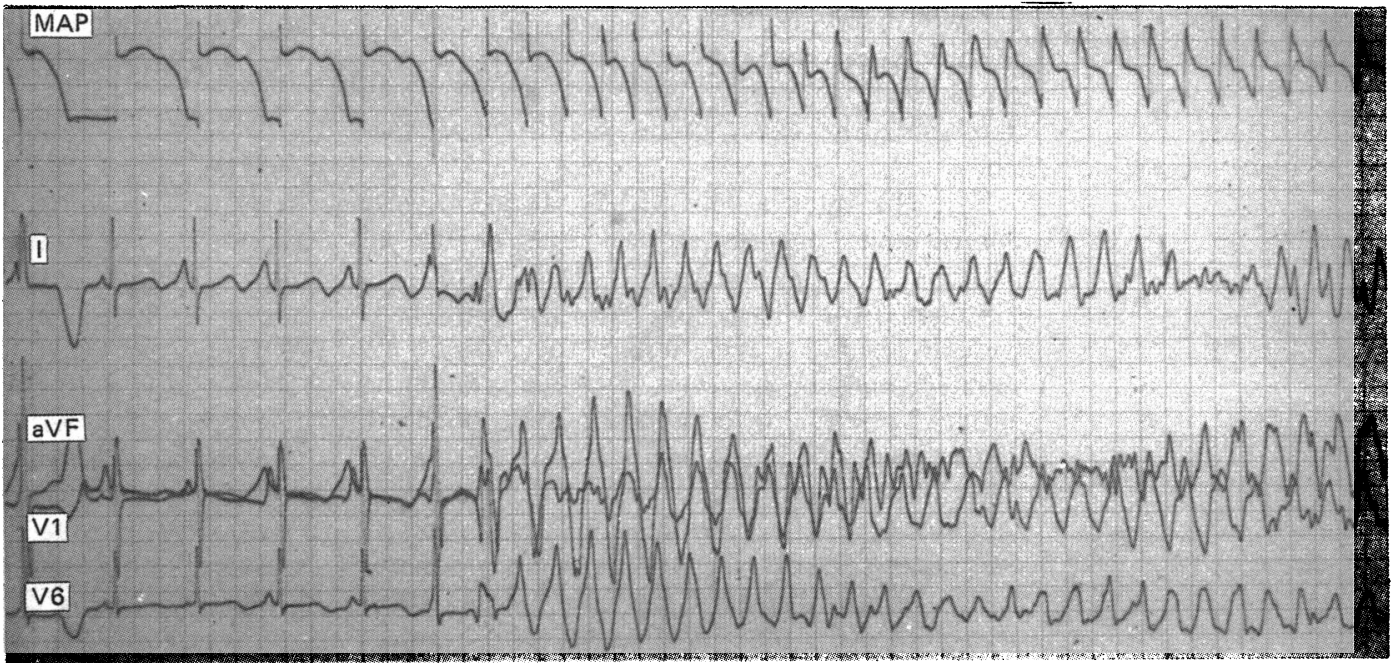


Figure 3 Rate adaptation of the $Q T$ interval in all eight patients with congenital long $Q T$ syndrome. For explanation see text.
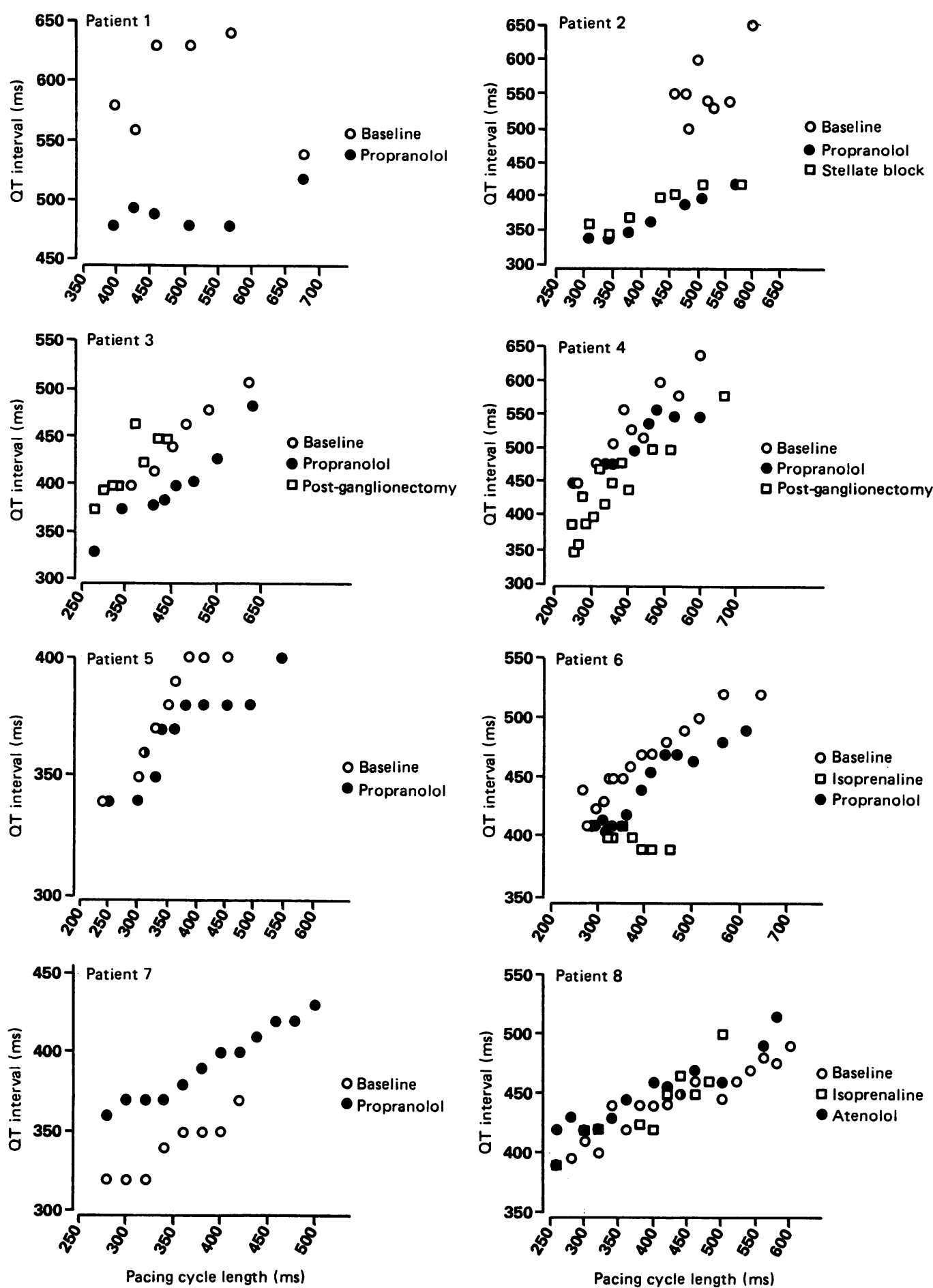

with no change in QT interval over the $130 \mathrm{~ms}$ (55 beats/minute) change in pacing cycle length. After the isoprenaline infusion this patient was given an injection of propranolol and her QT dynamics reverted to their normal baseline state.

After $\beta$ blockade there was no significant change in the paced QT intervals overall, although five patients (cases 1 to 3 and 5 and 6) showed a general shortening of their QT intervals, while patient 7 showed a lengthening of QT interval and patients 4 and 8 showed no change (fig 3). There was the same trend for the QT interval to shorten with increasing pacing rate and in particular patients 1 and 2 , who showed no rate adaptation in the baseline study, displayed appropriate QT interval shortening.

The two patients who underwent left stellate ganglionectomy (cases 3 and 4) had normal QT dynamics during their baseline studies and there was no change in either QT interval or QT dynamics after the operation. The one patient (case 2) who underwent left stellate block after the propranolol injection showed no additional change in QT dynamics or QT interval. In all three patients torsade de pointes degenerating to ventricular fibrillation was induced when the left stellate ganglion was manipulated surgically or with a needle. 
Figure $4 M A P_{90}$ rate adaptation in patients 1 and 4 to 8 . For explanation see text.
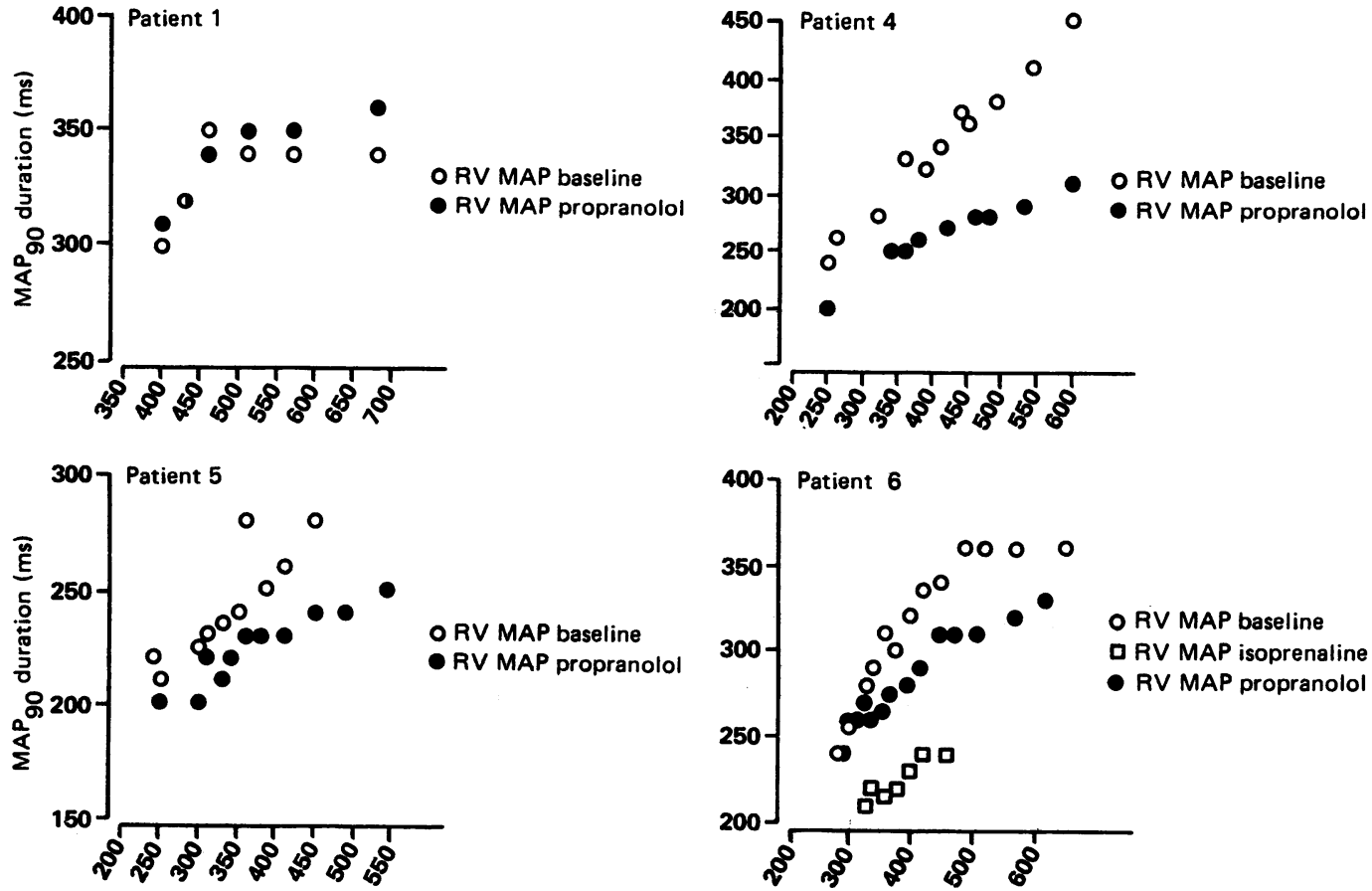

RV MAP baseline

O RV MAP baseline RV MAP propranolol

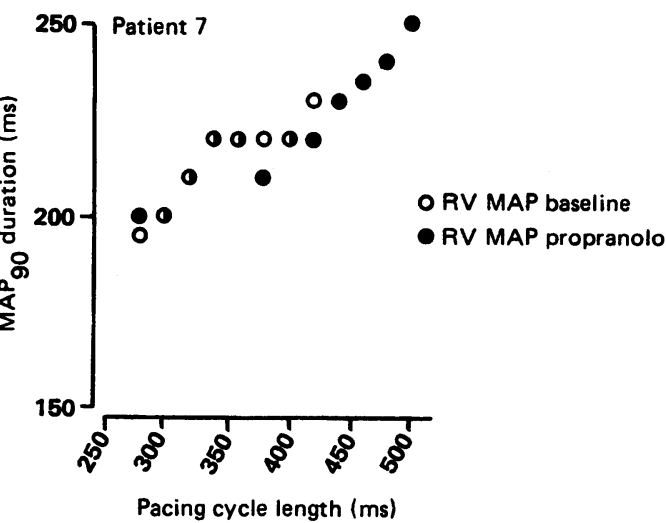

MONOPHASIC ACTION POTENTIAL RATE ADAPTATION

Ventricular monophasic action potentials were recorded during the ventricular pacing protocol in six patients (cases 1 and 4 to 8 ). In all patients, including those with altered QT dynamics, there was a decrease in $\mathrm{MAP}_{90}$ duration as the pacing rate was increased (fig 4). The range of decrease in $\mathrm{MAP}_{90}$ duration was variable - for example $50 \mathrm{~ms}$ in patient 1 (who had altered QT dynamics) and $210 \mathrm{~ms}$ in patient 4 (who showed normal QT dynamics). In the five patients in whom the study was repeated after propranolol (cases 1 and 4 to 7 ), there was no overall difference in $\mathrm{MAP}_{90}$ duration: three patients (cases 4,5 , and 6 ) showed shortening of the $\mathrm{MAP}_{90}$ duration, one patient (case 1) showed a slight increase in $\mathrm{MAP}_{90}$ duration, and one patient (case 7) showed no change. After propranolol in all patients, the monophasic action potential dynamics remained similar to the baseline study.

Two patients had the protocol repeated during an isoprenaline infusion. One (patient 6) showed a shortening of the $\mathbf{M A P}_{90}$ duration which was associated with $30 \mathrm{~ms}$ adaptation with increasing pacing rate (fig 4). This patient had no QT adaptation after isoprenaline. The other (patient 8) showed no change in $\mathbf{M A P}_{90}$

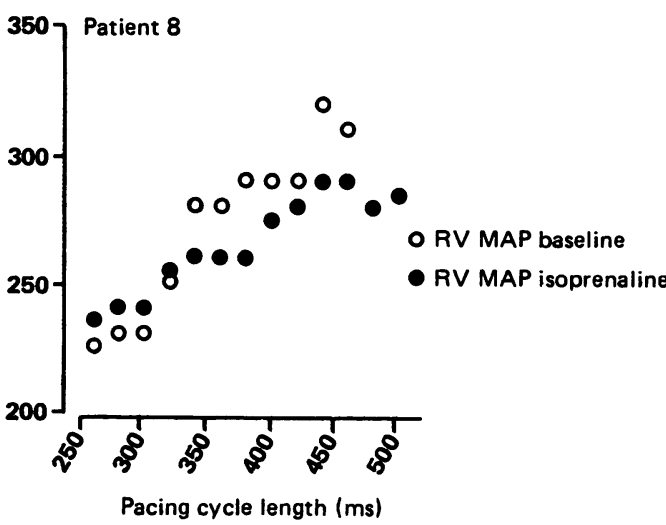

duration or dynamics after isoprenaline (fig 4). One patient (case 1) had simultaneous right apical and left apical ventricular monophasic action potentials recorded (fig 5 ). Whereas the left ventricular MAP $_{90}$ duration was less than the right ventricular $\mathrm{MAP}_{90}$ duration, both showed similar rate adaptation before and after propranolol. One patient (case 6) had right atrial monophasic action potentials recorded during atrial pacing (fig 6). Normal adaptation of the atrial $\mathrm{MAP}_{90}$ duration to increasing atrial pacing rate ${ }^{11}$ was seen in this patient.

DISPERSION OF MONOPHASIC ACTION POTENTIALS Dispersion of $\mathrm{MAP}_{90}$ duration was assessed in patients 6 and 8 by measurement of the right ventricular $\mathrm{MAP}_{90}$ duration at four and six sites respectively during atrial pacing at a cycle length of $500 \mathrm{~ms}$. In patient 6 the amount of dispersion was $50 \mathrm{~ms}$ ( 370 to $420 \mathrm{~ms}$ ) and in patient 8 it was $65 \mathrm{~ms}$ ( 315 to $380 \mathrm{~ms}$ ). In both patients the longest $\mathrm{MAP}_{90}$ durations were recorded from the right ventricular mid septum; however, the shortest $\mathrm{MAP}_{90}$ durations were recorded from the right ventricular inflow tract in patient 6 and from the free wall of the right ventricle in patient 8 . These values for dispersion are excessive compared with normal values. ${ }^{1213}$ 
Figure 5 Simultaneous right and left ventricular monophasic action potential recordings in patient 1 before and after propranolol.

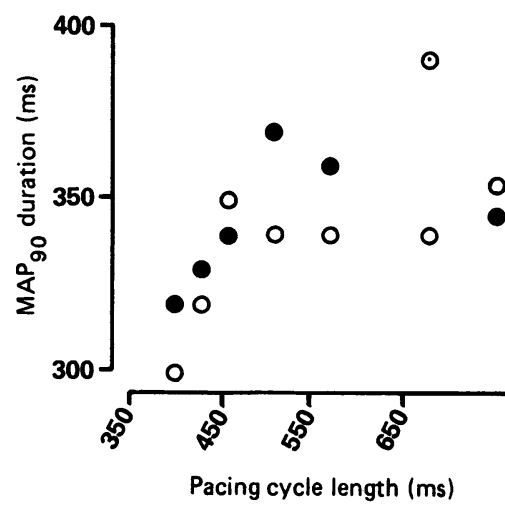

\section{Discussion}

The dynamics of the QT interval have been evaluated in normal subjects, but not in patients with congenital long QT syndromes. Studies in patients or subjects unaffected by prolonged QT syndromes showed that the QT interval is dependent on heart rate and also on changes in the catecholamine sensitivity of the heart. ${ }^{15}$ Other studies of changes in the QT interval with exercise showed an exponential relation between the QT interval and heart rate in normal subjects. ${ }^{16}$ Studies to evaluate the dynamics of the paced QT interval with atrial ${ }^{18}$ and ventricular pacing ${ }^{18} 19$ showed that in normal subjects or in patients with complete heart block the QT interval shortened with increasing pacing rate. The relation between these two functions was curvilinear: with a linear relation between heart rates of approximately 70 and 120 beats/minute..$^{20}$

Six of our eight patients with congenital QT prolongation showed an exponential or curvilinear pattern of QT rate adaptation. One (patient 2) of the two patients who showed no rate adaptation had a particularly severe manifestation of the syndrome with over 300 syncopal episodes. His resting QT interval was considerably prolonged (table 2) and he showed no rate adaptation (fig 3). It has been suggested that poor rate adaptation of the QT interval in patients with congenital long QT syndromes may increase the susceptibility to ventricular arrhythmias. ${ }^{4}$ The failure of QT rate adaptation was considered to be due to a reduction or lack of rate-related shortening of the ventricular action potential. Thus there is an increased likelihood that a ventricular extrasystole will occur in this vulnerable period and produce a ventricular arrhythmia. ${ }^{4}$ There

Figure 6 Right atrial monophasic action potential recordings during atrial pacing in patient 6.

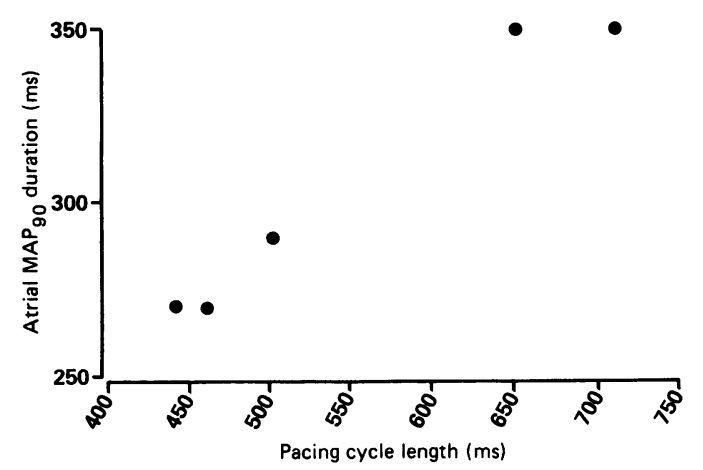

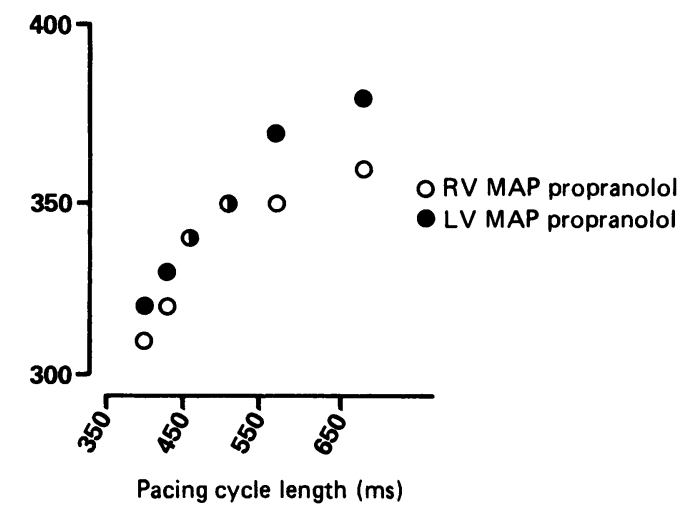

is, however, no direct evidence for this hypothesis. Only two of the seven patients with documented torsade de pointes did not have evident rate adaptation. However, an increasing ventricular rate may be associated with an increase in $U$ wave amplitude, a feature that often precedes the development of torsade de pointes. ${ }^{22}$ Changes in the $U$ wave amplitude, $T$ wave alternans, and the occurrence of torsade de pointes often appear shortly after an abrupt increase in heart rate, ${ }^{23}$ as in the present study, or can be elicited by other manoeuvres that increase sympathetic tone such as isoprenaline, as seen in patient 4 (fig 2 ), exercise, ${ }^{24}$ or the Valsalva manoeuvre. ${ }^{25}$

The recording of monophasic action potentials has provided useful insight into the mechanisms of congenital QT prolongation. Abnormalities of the monophasic action potential were described by Bonatti and colleages. ${ }^{10}$ These workers described "humps" arising from phase 3 of the action potential. These humps were regarded by others as representing early afterdepolarisations, ${ }^{22}$ a phenomenon produced in vivo by administering caesium chloride to canine hearts. ${ }^{26}$ In these studies ventricular arrhythmias with similar electrocardiographic characteristics to torsade de pointes were described which were initiated by early afterdepolarisations. Jackman and colleagues reported torsades de pointe arising in humans coincidentally with a hump on the endocardial monophasic action potential; and it has been suggested that early afterdepolarisations are responsible for the initiation of torsades de pointe in humans. In their illustration (fig $50,{ }^{22}$ ), the initiating ventricular extrasystole has a configuration and axis suggesting an origin remote from the site of the monophasic action potential recording. ${ }^{22}$ This was confirmed by the fact that the onset of the ventricular premature contraction preceded that of the right ventricular monophasic action potential. We too have noted this phenomenon (fig 2). Thus changes in local right ventricular monophasic action potential contour may be irrelevant to the onset of the premature extrasystole. Though we saw "humps" on the monophasic action potentials of patient 4 , it is clear that spontaneous torsade de pointes were not consequent upon the "hump" on the right ventricular monophasic action potential (fig 2 ).

The available data do not seem to support the early afterdepolarisations hypothesis, but they 
are consistent with the theory, advanced by Han and colleagues, Kuo and colleagues, and Surawicz that dispersion of repolarisation is responsible for torsade de pointes in these patients. ${ }^{27-30}$ When dispersion of repolarisation was assessed in patients with normal hearts the degree of monophasic action potential dispersion was up to $40 \mathrm{~ms}^{1213}$ and in patients with congenital QT prolongation it ranged from 100 to $270 \mathrm{~ms} .^{10}$ None the less, existing animal models of arrhythmias dependent on increased dispersion of repolarisation have been produced by large temperature gradients, ${ }^{29}$ or injections of large doses of contrast into conronary arteries, ${ }^{31}$ conditions seldom encountered in clinical practice. Nor does the production of early afterdepolarisations by caesium chloride occur in practice. Clinical observations of patients with torsade de pointes do not necessarily provide the solution to the question of mechanism, because most observations can be explained by both mechanisms. At the present time, neither of the two proposed substrates has been conclusively shown to be the cause of torsade de pointes in humans.

In normal subjects, the monphasic action potential duration decreased linearly with decreasing pacing cycle lengths over a range of 800 to $350 \mathrm{~ms} .{ }^{32}$ This has also been shown in guinea pig $^{33}$ and canine ${ }^{34}$ ventricles in vitro. It is of note that the decrease in monophasic action potential duration in our patients was more consistent than changes in QT adaptation. Because the $T$ wave is considered to be the summation of phase 3 of the action potential of all individual myocardial fibres, a parallel can be expected between the duration of the ventricular monophasic action potential and that of the QT interval. ${ }^{35}$ None the less, the precision of this relation is affected by individual variation in monphasic action potential duration as well as the precision of measurement of both functions, so it is difficult to interpret the discrepancy between these functions that was seen in two patients in our study. Furthermore, the monophasic action potential looks only at one site within the ventricular mass, whereas the QT interval is the summation of an infinite number and it is possible that if recordings were made from several sites, variability in monophasic action potential adaptation would be found, consistent with the abnormal QT changes. Unfortunately, the patient who had the least QT rate adaptation (case 2) did not have monophasic action potentials recorded.

$\beta$ Adrenergic blocking agents have long been used to treat arrhythmias associated with prolongation of the QT interval and were shown to reduce mortality over a ten year period from $78 \%$ in untreated patients to $6 \%$ in treated patients. ${ }^{36}$ Studies in which patients were given intravenous $\beta$ blockade have not shown any significant change in QT interval, ${ }^{37} 38$ although a tendency for the QTc value to shorten as the heart rate fell was noted. While our results did not show any overall change in QT interval during sinus rhythm after $\beta$ blockade, there was considerable interpatient variability, with some patients showing a shortening of QT interval and some an increase. It is of interest that propranolol normalised the QT rate adaptation in the two patients with abnormal baseline adaptation (patients 1 and 2 ). The mechanism of this normalisation is unclear but may be due to a reduction in the dispersion of repolarisation or a suppressant effect on afterdepolarisations, which has been shown in vitro. ${ }^{22}$

It is not surprising, in view of the small change seen in the QT interval, that no change in MAP $_{90}$ duration was noted in our patients after $\beta$ blockade. This accords with the work of others who also found no change in $\mathrm{MAP}_{90}$ duration after intravenous metoprolol. ${ }^{37}$ Others, however, found that long term treatment with atenolol or metoprolol increased the $\mathrm{MAP}_{90}$ duration by $12 \%$ in patients with coronary artery disease. ${ }^{37} 39$

There are some limitations in the present study with the evaluation of QT dynamics. Pacing for 10 seconds does not take into account hysteresis of the QT interval. We have previously shown that the decrease in QT interval with increasing pacing rate occurs in a bi-exponential form and takes approximately three minutes to complete $90 \%$ of its adaptation. ${ }^{40}$ If steady state QT intervals were obtained, however, the study would either be excessively long or only a few heart rates could be assessed. The pacing protocol was the same in all patients and it is surprising that no abnormalities were seen in the other six patients. It is unlikely that the observed abnormalities in rate adaptation were artefactual or due to inaccuracies in the measurement of the QT interval. All measurements were made by two observers, although in patient 1 the alteration of a few measurements would result in reasonably linear rate adaptation. For clinical reasons not all patients completed the full protocol.

In summary, in this study of ventricular repolarisation (monophasic action potential duration, monophasic action potential configuration ("humps"), monophasic action potential dispersion, and monophasic action potential/QT rate adaptation) in the long QT syndrome no single abnormality could be identified as being associated with the tendency to develop torsade de pointes. The meaning of "humps" or early afterdepolarisation seen in vivo remains unclear; when such features were seen in our patients they seemed to be irrelevant to the onset of the arrhythmia. Abnormal rate adaptation was found in only a few patients and is therefore unlikely to be directly related to the genesis of arrhythmias. Perhaps this response is found in only the most severely affected cases. Dispersion of repolarisation has long been suggested as a potential mechanism for ventricular arrhythmias in this syndrome. ${ }^{41}$ Our data, albeit limited, are not inconsistent with this suggestion but the mere presence of inhomogeneity of repolarisation during sinus rhythm cannot be adduced as evidence for the hypothesis. Our study has not clarified the role of differential sympathetic neural activity in this syndrome. ${ }^{36}$ Other evidence indicates that it is likely to be of secondary importance to a primary cellular 
abnormality that continues to exist when sympathetic influences are removed.

We thank Dr E A Shinebourne for permission to include patients $2-5$ in this study. NJL is the holder of a Squibb patients $2-5$ in this study. NJL is
Cardiovascular Research Fellowship.

1 Jervell A, Lange-Nielsen F. Congenital deaf mutism, prologned QT

2 Romano C, Gemme G, Pongiglione R. Aritmie cardiache rare dell'eta periatrica: II. Accessi sincopali per fibrillazione ventricolare parossistica. Clin Pediatr (Bologna) 1963;45:656-61.

3 Ward OC. New familial cardiac syndrome in children. $J$ Ir Med Assoc 1964;54:103-6.

4 Attwell D, Lee JA. A cellular basis for the primary long Q-T syndromes. Lancet 1988;i:1136-9.

5 Cowan JC, Yusoff K, Moore M, et al. Importance of lead selection in QT interval measurement. Am J Cardiol 1988;61:83-7.

6 Day CP, McComb JM, Campbell RWF. QT dispersion: an indication of arrhythmia risk in patients with long QT intervals. Br Heart J 1990;63:342-4.

7 Taran LM, Szilagyi N. The duration of the electrical systole (QT) in acute rheumatic carditis in children. Am Heart 1947;33:14-26.

8 Bazett HC. An analysis of the time relationships of the heart. Heart 1920;7:353-70

9 Ward DE. Prolongation of the QT interval as an indicator of risk of a cardiac event. Eur Heart J 1988;9(suppl G): $139-44$.

10 Bonatti V, Rolli A, Botti G. Recording of monophasic action potentials of the right ventricle in long QT syndromes complicated by severe ventricular arrhythmias. Eur Heart J 1983;4:168-79.

11 Brorson L, Olsson SB. Right atrial monophasic action potential in healthy males. Acta Med Scand 1976;199: p33-46.

12 Hoffman BF, Cranefield PF, Lepeschkin E, Surawicz B, Herrlich HC. Comparison of cardiac monophasic action potentials recorded by intracellular and suction elecpotentials recorded by intracellular and
trodes. Am J Physiol 1959;196:1297-301.

13 Franz MR, Bargheer K, Rafflenbeul W, Haverich A, Lichtlen $P$. Monophasic action potential mapping in human P. Monophasic action potential mapping in human subjects with normal electrocardiograms: direct evidence

of the genesis of the T wave. Circulation 1987;75:379-86.
14 Rickards AF, Norman J. Relation between QT interval and heart rate: new design of physiologically adaptive cardiac pacemaker. Br Heart $J$ 1981;45:56-61.

15 Horstmann E, Koenn B. Temporal relationship between exercise and $\mathrm{QT}$ shortening in patients with $\mathrm{QT}$ pace makers. PACE 1989;12:1080-4.

16 Lecocq B, Lecocq V, Jaillon P. Physiologic relation between cardiac cycle length and QT duration in healthy volunteers. Am J Cardiol 1989;63:481-6.

17 Romano M, Clarizia M, Onofrio E, et al. Heart rate, PR and QT intervals in normal children: a 24-hour Holter monitoring study. Clin Cardiol 1988;11:839-42.

18 Milne JR, Camm AJ, Ward DE, Spurrell RAJ. Effect of intravenous propranolol on QT interval. A new method of assessment. Br Heart $J$ 1980;43:1-6.

19 Milne JR, Ward DE, Spurrell RAJ, Camm AJ. The exercise. PACE 1982;5:352-8.

20 Susmano A, Graettinger JS, Carleton RA. The relationship between Q-T interval and heart rate. $J$ Electrocardiol 1969;2:269-74.
21 Akhras F, Richards AF. The relationship between QT interval and heart rate during physiological exercise and pacing. Jpn Heart J 1981;22:345-51.

22 Jackman WM, Friday KJ, Anderson JL, Aliot EM, Clark M Lazarra $R$. The long QT syndromes: a critical review, new clinical observations and a unifying hypothesis. Prog Cardiovasc Dis 1988;31:115-72.

23 Wellens HJJ, Vermuelen A, Durrer D. Ventricular fibrilla tion occurring on arousal from sleep by auditory stimuli. Circulation 1972;46:661-5.

24 Phillips J, Ichinose $\mathrm{H}$. Clinical and pathologic studies in the hereditary syndrome of a long QT interval, syncopal spells, and sudden death. Chest 1970;588:236-43.

25 Mitsutake A, Takashita A, Kuroiwa A, Nakamura $M$ Usefulness of the Valsalva maneuver in management of the long QT syndrome. Circulation 1981;63:1029-35.

26 Levine JH, Spear JF, Guarnieri T, et al. Cesium chlorideinduced long QT syndrome: demonstration of afterdepolarizations and triggered activity in vivo. Circulation 1985;72:1092-103.

27 Han J, Goel BG. Electrophysiologic precursors of ventricular tachyarrhythmias. Arch Intern Med 1972 192:749-55.

28 Han J, Moe GK. Nonuniform recovery of excitability in ventricular muscle. Circ Res 1964;14:44-60.

29 Kuo CS, Munakata K, Reddy CP, Surawicz B. Characteristics and possible mechanism of ventricular arrhythmi dependent on the dispersion of action potential durations. dependent on the dispersion of

30 Surawicz B. Electrophysiologic substrate of torsade de pointes: dispersion of repolarization of early afterdepolarizations? J Am Coll Cardiol 1989;14:172-84

31 Murdock DK, Euler DE, Becker DM, Murdock JD, Scan lon PJ, Gunnar RM. Ventricular fibrillation during coronary angioplasty: an analysis of mechanisms. Am Heart 1985;109:265-73.

32 Franz MR, Swerdlow CD, Liem B, Schaefer J. Cycle length dependence of human action potential duration in vivo. Effect of single extrastimuli, sudden sustained rate acceleration and deceleration, and different steady-state frequencies. J Clin Invest 1988;82:972-9.

33 Savigny LV, Hohnloser S, Antoni H. Effects of changes in frequency on guinea pig ventricular action potential duration and on QT interval under different experimental conditions. Basic Res Cardiol 1981;76:276-88.

34 Moore EN, Preston JB, Moe GK. Durations of transmembrane action potentials and functional refractory periods of canine false tendon and ventricular myocardium. Comparisons in single fibres. Circ Res 1965;27:259-73.

35 Olsson SB, Brorson L. Edvardson N, Varnauskas E. Estimation of ventricular repolarization in man by monoEstimation of ventricular repolarization in man by monophasic action potential

36 Schwartz PJ, Locati E. The idiopathic long QT syndrome: pathogenetic mechanisms and therapy. Eur Heart pathogenetic mechanism

37 Edvardsson N, Olsson SB. Effects of acute and chronic beta receptor blockade on ventricular repolarisation in man. $B$ Heart $J$ 1981;45:628-36.

38 Creamer JE, Nathan AW, Shennan A, Camm AJ. Acute and chronic effects of sotalol and propranolol on ventricula repolarisation using constant-rate pacing. $\mathrm{Am} \mathrm{J}$ Cardio 1986;57:1092-6.

39 Way BPJ, Forfar JC, Cobbe SM. Comparison of the effects of chronic oral therapy with atenolol and sotalol on ventricular monophasic action potential duration and effective refractory period. Am Heart $J 1988 ; 116: 740-6$.

40 Lau CP, Freedman AR, Fleming S, Malik M, Camm AJ, Ward DE. Hysteresis of the ventricular paced QT interva in response to abrupt changes in pacing rate. Cardiovasc Res 1988;22:67-72.

41 Surawicz B, Knoebel SB. Long QT: good bad or indifferent. J Am Coll Cardiol 1984;4:398-413. 